\title{
Controllers using Discrete Sliding Mode Control for $1 \&$ 2-link FMs with Output Samples
}

\author{
Joshi Shubangi Milind, Arunkumar G., T.C.Manjunath
}

\begin{abstract}
The main highlight of this paper being, the design \& development of Controllers using Discrete Sliding Mode Control for 1 \& 2-link FMs with output samples is being presented for controlling a 2-link FRM considering 2 DOF in 3 Dimensional Euclidean space along with the simulation results. A 2-link FRM with 2-DOF is being considered and the base motor is attached to the single flexible link \& the other link is fixed to the extreme end of the 1st link to which the shoulder motor is connected and the entire set-up is a single structure one and 2 motors are being used for actuation purposes \& is being controlled / regulated using the developed sliding mode controller, i.e., the dual links are rotating with respect to the base, further the link-2 is rotating with respect to the shoulder axes \& the joint axes of the two are parallel and perpendicular w.r.t. the robot work surface. Hence, the entire system can be called as a planar mechanism, i.e., the 2 links are moving parallel to the plane of the work surface $(x-y$ plane). There are 2 joints, viz., base joint \& the shoulder joint along with the end-effector being the tip of the 2-link manipulator. The small errors that occurs in the control signal $u$ is due to the changes during the set-point. Simulink model is being developed in the Simulink-Matlab environment \& after running the model, the simulation results observed, the obtained results shows the efficacy of the methodology developed.
\end{abstract}

Keywords-Robots, Flexible, Manipulators, 3D, Planar, Motor, Sliding Mode Control, Simulink, Matlab, Simulation, Run time, Results.

\section{INTRODUCTION}

A brief introduction about the controller design using the discrete sliding mode control theory from the output samples is dealt in this paragraph with aftermath being used to control the various parameters of the 1-link \& 2-link flexible manipulator. Once, the controller is designed using the sliding mode concepts, the proposed controller when it is connected in-loop with the plant model (1-link or 2-link flexible manipulator) and the control strategy developed is tested for its effectiveness. Matlab tool is being used to develop the control algorithm, apply it to the flexible system and then to observe the simulation results. The research work done is compared with some of the works of the yesteryears. The simulink model for the DSMC designed controller using the output samples is also developed \& the simulation results are observed for both the cases of the flexible manipulator. The

Revised Manuscript Received on July 22, 2019.

Joshi Shubangi Milind, Ph.D. Research Scholar-Part Time, Dept. of ECE, VTU RRC-Belgaum, Karnataka

Dr. Arunkumar G., Associate Professor \& Head, ECE Dept., JSS Academy of Tech. Education (JSSATE), Noida, Uttar Pradesh

Dr. T.C.Manjunath, Professor \& Head, ECE Dept., Dayananda Sagar

College of Engg. (DSCE), Bangalore, Karnataka

Email : dr.manjunath.phd@ieee.org paper concludes with the simulated results discussion with a brief conclusion about the proposed research work undertaken in this paper.

The organization of this research paper is done in the following manner. A brief introduction to the paper is depicted in the first section, followed by a brief insight into the development of the DSM Control Design using o/p Samples for FMs in the section II. Next, the mathematical modelling development is depicted in the section III. The entire control design for Single-link FM \& a dual link FRM is presented in subsequent sections IV \& V respectively one after the other. The development of the DSMC simulink model is presented in section VI. The section VII finally concludes the paper followed by an exhaustive list of references.

\section{A BRIEF INSIGHT INTO THE DEVELOPMENT OF THE DSM CONTROL DESIGN USING O/P SAMPLES FOR FLEXIBLE MANIPULATORS}

Variable structure (VSC) frameworks showing sliding mode movement has been explored since long from the seasons of Gao, who was the pioneer in this field.
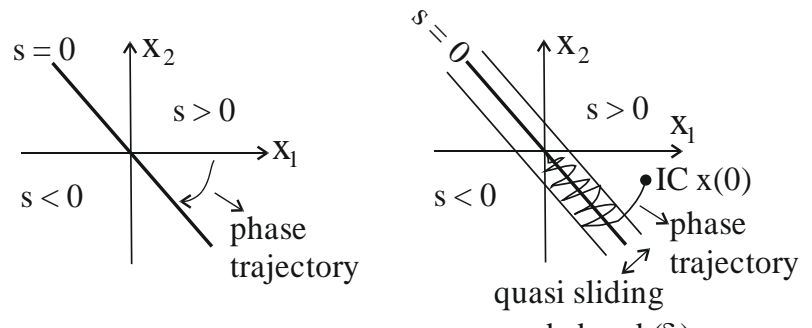

mode band $(\delta)$
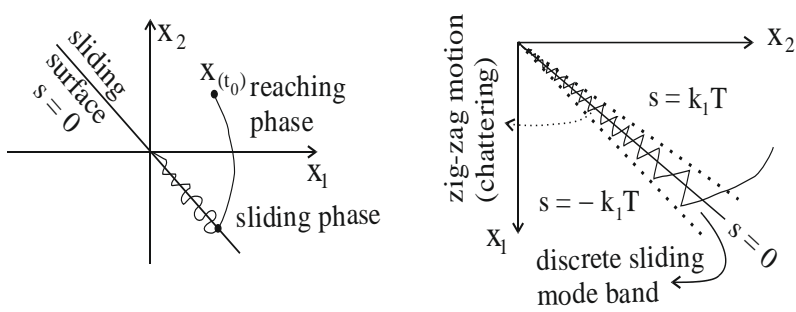

Fig. 1 : SMC \& the DSMC concepts showing chattering

Because of the simple structure of the sliding mode controller, they are used in different applications such as power converter, disk drives servos, robot manipulator, induction motor, stepper motor control and for a host of automobile control applications, etc.

Due of the advent of computers, this computer implementation of SMC has motivated the research with respect to the DSMC 
algorithms. The DSMC has been investigated since 1950's. The frequency of switching in SMC can't surpass the $f_{s}$ (samp. freq.) in light of the fact that the control $\mathrm{i} / \mathrm{p} u$ is constantly held steady between the sequential sampling times. Because of this limitation, it results in chattering in discrete sliding mode control.

Hence, a concept known as quasi sliding mode became popular due to the chattering effect (which is a zig zag like motion). The sliding mode, discrete sliding mode, band, chattering, etc. can be best understood from the Fig. 1. The chattering phenomenon, which is the inherent property of any system is always present \& cannot be avoided, but can be limited. The important point is that in quasi sliding mode the sliding mode motion cannot be confined on the sliding manifold and hence the invariance properties found in continuous time sliding mode does not hold for its discrete counter-part. Utkin says, in case of discrete variable structure system, the quasi sliding mode motion is possible as the action of control effort can be impeded only at the appropriate sampling instants. This inherently introduces a chattering known as discretization chatter and the system motion confines to a band known as the QSM band as shown in the Fig. 1.

Gao proposed a strategy for DSMC utilizing the methodology of the achieving law (RLA). This achieving law guarantees that the framework state direction will hit the exchanging complex and experience a crisscross like movement, along these lines bringing about a semi sliding mode movement, about the exchanging complex lastly stays inside a band called as the "Semi Sliding Mode Band (QSMB)". As indicated by the idea proposed by Utkin and Drakunov, the framework demonstrates sliding mode conduct if their movement is limited to an exchanging complex in the state space that will be come to in a limited time. At that point, a discrete time sliding mode control may emerge in the framework with piecewise consistent control $u$. In this context, an important and interesting possibility for discrete processing in discrete sliding mode had been proposed by Milosav in his research paper.

Versatile sliding mode control (also called as adaptive SMC) for DT-LTI which ensures that the perfect sliding starts after a limited interval of time was presented by Utkin in a number of research papers \& used it for a variety of applicational problems. Robust DTSMC based on the above control was been proposed by Dra, which showed robustness to parametric variations. The main rules for the presence of DTSMC and for the control law which takes out the criss-cross movement of the DTSM was been exhibited in Yu's research paper.

Another robust DT chattering free algorithm was proposed by Milosevic, which showed that the state trajectory reaches nearer to the manifold of the sliding state/surface in definite (fixed) tines using the non-linear control \& then it reaches to the sliding manifold in one single step using an appropriate linear control law $u(k)$. Hence, discrete sliding mode motion without chattering was obtained. It was shown that in the presence of the disturbances, the system motion confines to a small band around the sliding manifold.
The majority of the sliding mode control issues require a full-state $\mathrm{f} / \mathrm{b}$. In any case, in real time apps and in some perfect circumstances, the estimation of all the states of the DT/CT system may be neither conceivable nor attainable, i.e., can't be completely quantifiable (cant be measured at all). Since the $\mathrm{o} / \mathrm{p}$ of the system is accessible, $\mathrm{o} / \mathrm{p} \mathrm{f} / \mathrm{b}$ could be utilized to structure the controller (this is what is called as output feedback control) as output of the system is always available as once you give the input, we get the output, so, make use of this output $y$. Recently DSMC \& robust DSMC which results in quasi sliding mode motion was proposed by couple of authors using fast output sampling feedback techniques, which used the output of the system $y$.

The famous four authors DTSMC algorithm proposed by Thakkar, Saaj, Janardhanan \& Bandyopadhyay on the stepper motor design of the controlling using the concepts of MROF SMC concept has been used by many researchers across the wold for various applications. In our research work considered, the famous 4 author algo is being used to control the 1-link \& 2-link flexible robotic manipulator for the end-effector precise control. Also, the authors had presented a chattering reduction algorithm which is accomplished using the DT power rate reaching law and the concepts of MROF-multirate output feedback.

It was shown that with appropriate choice of controller parameter, the fast reaching to the set state with less chattering can be obtained \& thus provided a great breakthrough in the sliding mode approach. In another algorithm which they had presented, a reaching law was modified by developing a controller having 2 parts, viz., a non-linear part and a linear part. If the non-linear parts brought the state trajectory into the nearness of the manifold of the sliding zone $\&$ the linear part used to obtain the motion of the sliding along the "sliding-manifold" just in 1 iterational step as a result of which the chattering was removed to a maximum extent. The authors also discussed the issues of uncertainties by taking a number of numerical examples of state space models of different sizes.

Control of FRMS finds a ton of uses in the cutting edge world, especially in the field of avionics, robotics \& the smart intelligent flexible systems. The need for a sophisticated control arises from the discrete sliding mode concepts, which has gained a lot of advantages over the traditional control schemes for controlling the various parameters of the plants. One such SMC algo is the DSMC algo often called as the Discrete-Sliding-Mode-Control. The output of the plant, i.e., the 'displacement', velocity, acceleration (each one obtained by the differentiation of the earlier ones) is considered as the parameter for control purposes, in our case, the displacement is being considered as the control variable to be tracked upon with. In the following paras, a exhaustive summary of the DSMC is interpreted which can be understood in a simple manner by any researcher.

This special discrete control algorithms' developed to cater for the flexibility in the robotic arms and these algorithms are not that complex, considering that the system is non-linear as generally all flexible systems even though they are non-linear will be 
linearized about an operating point. In the research work considered, DSMC is used for controlling the dynamic motion of the $n$-link FRMs \& take the tip to a specified location and also to track the set-points, curb down the vibrations with less errors. To design the controller, a state space model is obtained first from the first principles starting from the $\mathbf{M}, \mathbf{B}$, K parameters. 2 separate cases of the flexible robotic manipulator are presented here with, viz., the SISO (1-link) control, second-the MIMO (2-link) control, in the sense the former has one actuator - so, 1 input \& 1 output, whereas the latter has two actuators - so, 2 inputs \& 2 outputs.

Open loop (OL) \& closed loop (CL) responses are also observed with the gain of the DSMC. Simulations are carried out with \& without the DSMC controller to exhibit the authenticity w.r.t. the different strategies of control by comparing with the work done by other researchers [21] \& [33]. The DSMC control algorithm actually needs the plant system to be completely controllable \& be observable, thus giving the desired performances even in the presence of un-measured disturbances. The performance of the DSMC controller will be tested on the $1 \& 2$ link flexible manipulator $\&$ could be generalized for a $n$-link flexible manipulator case also, which could be treated as future works.

The SMC hypothesis is really created on the idea of changing the structure of the controller because of the changing state $\mathrm{x}$ of any plant model of the system in such a way that the desired response $y$ is obtained, the SMC theory being proposed by Utkin. Hung \& Young said, " $a$ high speed switching control action can be used in order to switch between different structures \& the trajectory of the given system can be forced to move along a chosen manifold in the state space". This being called as the exchanging complex. The conduct of the CLCS is in this way controlled by utilizing the SMC's sliding surface. Till date, a large number of researchers have put forth lot of efforts in studying the Discrete Sliding Mode (DSM) and quite a number of controller designs have been put forward by many authors \& all those control laws were used for a variety of applications, to name a few of them, Gao, Furuta, Werner, Bag.

In the event that the discrete sliding mode is considered, the control activity is actuated at testing moments \& $u$, the control exertion is treated as consistent over each inspecting period $\tau$. At the point when the state $x$ achieves the exchanging surface, the subsequent DT switching's cannot maintain the state on the sliding surface. Since the DSM undergoes only quasi-sliding motion, the famous sliding mode specialist, Gao introduced a new law called as the 'reaching-law approach' to design the proposed controller for any DT system using SFB. This achieving law completely guaranteed that the state trajectory of the system will hit the vicinity of the manifold of switching and from there on will experience a crisscross movement about the manifold of the switching zone and achieve the harmony (stable) point.

The extent of each of the repetetive zig-zagging step/s goes on decreasing in order to make the state trajectory to stay in a pre-specified sliding band. This specified switching band is called the QSM band. Many of the SMC methods requires full $\mathrm{SFB}$.

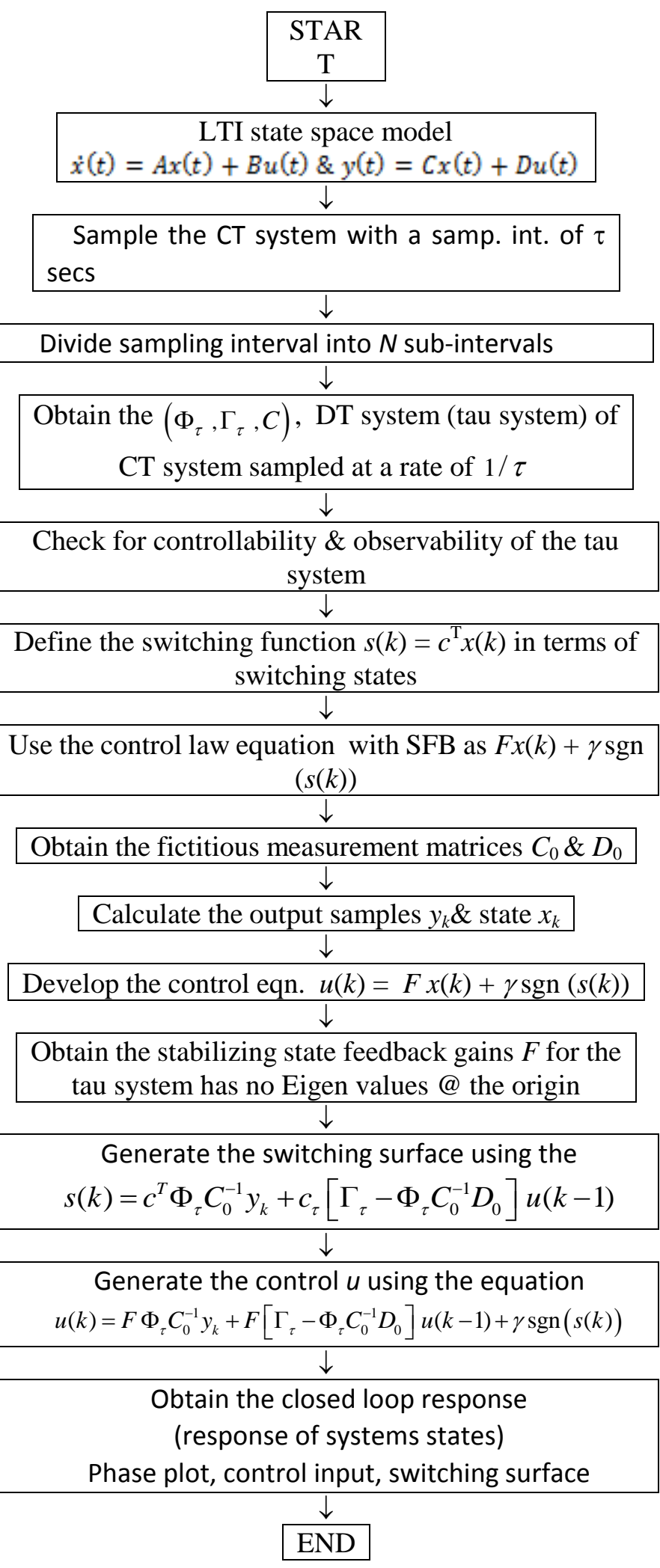

Fig. 2 : Proposed flow-chart for the controller design using the DSMC concepts

But in some of the ideal \& practical situations, it is a generalized concept that many of the states of the system will be available for making measurements and hence the control $u$ may or may not be feasible. Since the system output is always available (any system has 3 important parts - input, system, output), the $\mathrm{o} / \mathrm{p}$ of the control 
system can be feedback \& this output $y$ can be used to design the controller to control a particular variable or a group of variables, say $\theta, \omega, \& \alpha$, i.e., angular displacement, angular velocity and angular acceleration.

Considerable research work in this exciting area has been carried out in the field of output feedback SMC. The static o/p $\mathrm{f} / \mathrm{b}$ (SOFB) problem is one of the most well-researched problems in majority of the control theory \& its examples which makes use of the theory. But, still now, there are no good results which could be seen till date \& this has led us to the extent that the complete assignment of the poles in the $\mathrm{p}-\mathrm{z}$ plane is possible using static $\mathrm{o} / \mathrm{p} \mathrm{f} / \mathrm{b}$. Because of this reason, any control engineer can shift to the static o/p f/b as the output of the system is readily available.

In this unique circumstance, the yield criticism can be acknowledged utilizing quick yield inspecting ideas created by Werner and Furuta. Werner has utilized the quick yield inspecting (FOS) criticism which has the highlights of static yield input and makes it conceivable to discretionarily allocate the framework posts. In contrast to static yield criticism, quick yield testing input dependably ensures the soundness of the shut circle framework. Recently a FOS SMC algorithm was put forward by Chakravarthini Saaj, which uses reaching law approach. The output is corrected before being used for feedback purpose.

The o/p f/b gain $L$ is obtained from the SFB gain $F$ using the mathematical linearity equation $L C=F$ as given by Werner. An improvement of the technique was proposed by Saaj, where output correction will not be required to generate the control signal $u$. The method proposed by Saaj does not need the calculation of the output feedback gain $L$ using the previously mentioned relation. In this unique circumstance, the exchanging capacity $\mathrm{s}(\mathrm{k})$ and control input $\mathrm{u}$ is straightforwardly acquired as far as the quick past control esteems $u$ and the past yield tests $y$.

\section{MATHEMATICAL MODELLING DEVELOPMENT}

The proposed flow-chart for the controller design using the discrete sliding mode concept using output samples is depicted in the Fig. 2. Now, to implement this proposed flow-chart shown in the Fig. 2 for the controller design using the DSMC concepts on the 1-link \& 2-link flexible robotic manipulator. A Linear Time Invariant state space plant model with as shown in eqn. (1) is modelled as

$$
\begin{aligned}
& \dot{x}(t)=A x(t)+B u(t) \\
& y(t)=C x(t)+D u(t)
\end{aligned}
$$

where

$$
x \in R^{n}, u \in R^{m}, y \in R^{p}, A \in R^{n \times n}, B \in R^{n \times m}, C \in R^{p \times n}
$$

, $A, B, C$ are numerical matrices and it can be assumed that the pair $(A, B)$ is controllable and the pair $(C, A)$ is observable. It is to be noted that $A, B, C, D, x, u, y$ are the system matrix, $\mathrm{i} / \mathrm{p} \& \mathrm{o} / \mathrm{p}$ matrices, transmission matrix, state variable, $\mathrm{i} / \mathrm{p} \& \mathrm{o} / \mathrm{p}$ variable of the plant (1-link \& 2-link flexible system) \& are constant matrices of requisite dimensions. Consider the discrete system obtained using the sampling of the CT system by $\tau$ units

$$
\begin{gathered}
x(k+1)=\Phi_{\tau} x(k)+\Gamma_{\tau} u(k) \quad \& \\
y(k)=C x(k)
\end{gathered}
$$

Here, the parameter $\tau$ is the sampling period, $x$ is $n$-dimension state vector, $u$ is scalar, $y$ is output vector, \& the matrices $\Phi_{\tau}, \Gamma_{\tau}$ and $C$ are of appropriate dimensions. Here, we make an assumption that the matrix pairs $\left(\Phi_{\tau}, \Gamma_{\tau}\right) \&\left(\Phi_{\tau}, C\right)$ are controllable \& observable. An achieving law for the SMC of a DT framework as developed by Gao, which has the equation of the following form $\&$ is given by

$$
s(k+1)-s(k)=-q \tau s(k)-\varepsilon \tau \operatorname{sgn}(s(k))
$$

where $\tau>0$, is the period of sampling, $\varepsilon>0, q>0,(1-q \tau)>$ 0 and $s(k)$ is the switching function defined as a function of the system states as

$$
s(k)=c^{T} x(k)=0
$$

Using the reaching law given in the equation (3), the control law $u(k)$ for the control system model as given by the equation (2) has been developed as per Gao with the control model

$$
u(k)=F x(k)+\gamma \operatorname{sgn}(s(k))
$$

Here,

$$
\begin{gathered}
F=-\left(c^{T} \Gamma_{\tau}\right)^{-1}\left[c^{T} \Phi_{\tau}-c^{T} I+q \tau c^{T}\right] \\
\gamma=-\left(c^{T} \Gamma_{\tau}\right)^{-1} \varepsilon \tau
\end{gathered}
$$

Bartoszewicz proposed the quasi-sliding mode band's width $\delta$ as

$$
2 \delta \leq \frac{2 \varepsilon \tau}{2-q \tau}
$$

It is to be noted that the system states like within the steady state band given by the above band's width $\delta$ equation. FOS technique was dealt with in brief in the work done by the same authors, i.e., by us (concept introduced in another paper). In this method, the $\mathrm{o} / \mathrm{p} \mathrm{f} / \mathrm{b}$ law is utilized to understand a discrete state input gain by multirate perceptions of the $\mathrm{o} / \mathrm{p}$ signal and the control $\mathrm{i} / \mathrm{p}$ is held steady amid each testing period $\tau \&$ this concept is being clubbed with the DSMC to obtain a revised type of control called as the FOS-SMC with output samples as proposed by the famous 4-author algorithm (Thakkar, Saaj, Janardhanan, Bandyopadhyay), a small review of which is presented as follows.

Here, the reaching law approach as proposed by Gao, where the control takes the form as mentioned in equation (5) is being used with the value of the $\gamma$ as defined in the equation (7). Next the control law $u(k)$ is obtained as follows after applying to the discretized system in equation (2) as

$$
x(k+1)=\left(\Phi_{\tau}+\Gamma_{\tau} F\right) x(k)+\Gamma_{\tau} \gamma
$$




$$
y_{k+1}=\left(C_{0}+D_{0} F\right) x(k)+D_{0} \gamma
$$

Using the equation no. (9), it could be reduced further as $x(k)=\left(\Phi_{\tau}+\Gamma_{\tau} F\right)^{-1} x(k+1)-\left(\Phi_{\tau}+\Gamma_{\tau} F\right)^{-1} \Gamma_{\tau} \gamma(11)$

Now from the equation (11), substitute for $x(k)$ from equation (10) \& further simplifying all those relations, we get the relation between the states $\&$ the output samples $y_{k}$ as and simplifying them gives the relation between output samples $x(k)$ and $y_{k}$ and state $x(k)$ as

$$
y_{k}=\mathbf{C} x(k)+\alpha \quad \& \quad \alpha=\left(D_{0}-\mathbf{C} \Gamma_{\tau}\right) \gamma
$$

When $x(k)$ is substituted from the equation (12) into equation (5), we get the output feedback control law, which does not need the output correction as given by Saaj \& Bandyopadhyay as given by the equation (13) in

$$
u(k)=L y_{k}+\eta(k) ; \text { where } \eta(k)=-L \alpha+\gamma(13)
$$

The above-mentioned control $u$ drives the system states $x$ towards the sliding manifold \& by the time the trajectory of the state will cross the line of switching, then the control $u$ will assume the form

$$
u(k)=L y_{k}+\eta(k)
$$

where $\eta(k)$ switches between the values given by $\eta_{1}=L \alpha+\gamma$ and $\eta_{2}=-(L \alpha+\gamma)$ at every subsequent sampling instant $\tau$. Finally, the relation for switching function $s(k)$ in terms of $\mathrm{o} / \mathrm{p}$ is given by

$$
s(k)=c^{T} \mathbf{C}^{-1}\left(y_{k}-\alpha\right)
$$

Now, in order to determine the mathematical model for the SMC using the output samples, consider the discretized CT system by $\tau$, i.e., the tau system with the value of $k=0$ as given by the equation (16) as

$$
x(\tau)=\Phi_{\tau} x(0)+\Gamma_{\tau} x(0) \quad \& \quad y_{\tau}=C_{0} x(0)+D_{0} u(0)
$$

Now, at this juncture, the o/p samples are not seen before the time of $t=0 \&$ therefore the control signal can be deduced using the eqn. (5) by putting $k=0$, thus the switching can be deduced as finally

$$
s(\tau)=c^{T} \Phi_{\tau} C_{0}^{-1} y_{\tau}+c^{T}\left(\Gamma_{\tau}-\Phi_{\tau} C_{0}^{-1} D_{0}\right) u(0)
$$

$\&$ the control at $\tau$ is given by

$$
u(\tau)=F \Phi_{\tau} C_{0}^{-1} y_{\tau}+F\left(\Gamma_{\tau}-\Phi_{\tau} C_{0}^{-1} D_{0}\right) u(0)+\gamma \operatorname{sgn}[s(\tau)]
$$

Since it can be presumed that $n=N$ as the proposed system is completely observable, i.e., $C_{0}^{-1}$ exists. Finally, after simplification, a generalized expression for the switching function \& control as

$$
s(k)=c^{T} \Phi_{\tau} C_{0}^{-1} y_{k}+c^{T}\left(\Gamma_{\tau}-\Phi_{\tau} C_{0}^{-1} D_{0}\right) u(k-1)(19)
$$

From the above equation of $u(k)$, a generalized statement can be made, i.e., the states of the system may not be needed for the evaluation of the switching function or for the purpose of feedback. This concept is being used in our work to control the 1-link \& 2-link flexible robotic manipulator's some of the system parameters such as the position, etc.

\section{CONTROL DESIGN FOR SINGLE LINK FLEXIBLE MANIPULATOR}

It should be noted that in this case, a single flexible link is connected to the hub where a single actuator is used for actuation purpose. Refer Figs. 15-17 (appendix) for the diagrammatic representation of the flexible system with its specifications in Table 1 (appendix). The actuator is actuated upon by a control effort $u$ such that the desired set-point is reached. In the sense, when the single link gets actuated, the motor as well as the flexible link starts vibrating.

At the tip of the flexible link, one displacement sensor is placed, which is used to give the feedback to the system and could be used for tracking purpose. This displacement $x(\theta)$, which is nothing but the output of the system $y$ could be considered as one of the state variable, which is used for tracking and bringing back to the desired position in no time. In other words, there is one actuator (motor)-input \& one sensor (used to obtain the displacement of the end-effector-output as such the 1-link flexible system can be considered as a SISO system.

Simulation study has been carried out for a particular designed set values of the DSMC parameters $q, \tau, N, \Delta, \varepsilon$. The simulation results can also be observed for different sets of parameters of $q, \tau, N, \Delta, \varepsilon \&$ computing the switching surfaces $s(k)$ along with the control inputs $u(k)$, which can also be observed that the responses of the states $x_{1}, x_{2}$ will converge to zero (0) from a given IC $x(0)$. Also, the phase plots for the flexible system shows that the states of the system exhibiting the quasi-sliding motion. Another reaction plot demonstrates that the exchanging capacity diminishes towards zero from the underlying worth and remain inside a little band in the area of the exchanging line. Since the single-link model is modelled as a $(2 \times 2)$ case, there will be only 2 states $x_{1} \& x_{2}$ as the size of the $A$ matrix will be of $(2 \times 2)$. The values chosen for the tracking control were

$$
q=1, \tau=0.2, N=10, \Delta=0.1, \varepsilon=0.005 .
$$

From the simulation results, it can be clearly observed that the set-point tracking control is good. Matlab tool has been used to develop the . $m$ code. The developed algorithms are written in the fogm of a code, the code is run and the various responses that were considered in the sim study were observed, the results were discussed. The following parameters were observed from the simulation results.

- State trajectory

- Phase plots

- Output tip displacements

- System states

- Switching function 
- Control input

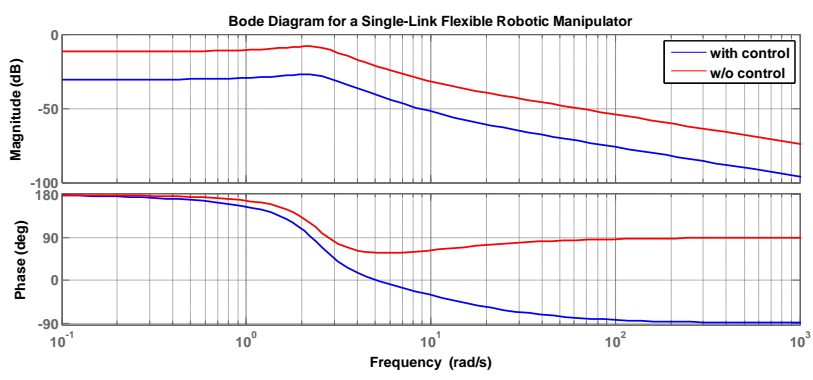

Fig. 3 : Bode diagram (frequency plot) for a one link-FRM showing with \& w/o control

From the simulation results of the various previous parameters, it can be concluded that the effectiveness of the proposed algorithms are observed as they reach the stability point in no time (just within 5 seconds). The frequency response plot also shows how the effect has taken place before $\&$ after the control effect. The control effect taken is also less as it is a 1-link manipulator case.
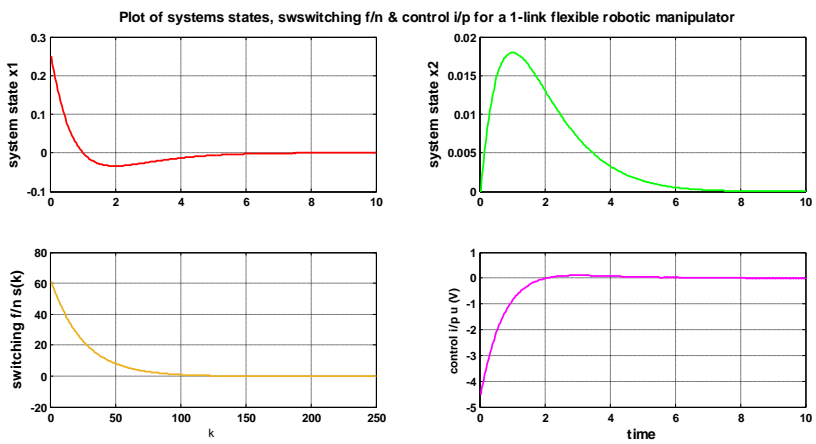

Fig. 4 : System responses (plot of the system states), switching function \& control input for a one link FRM

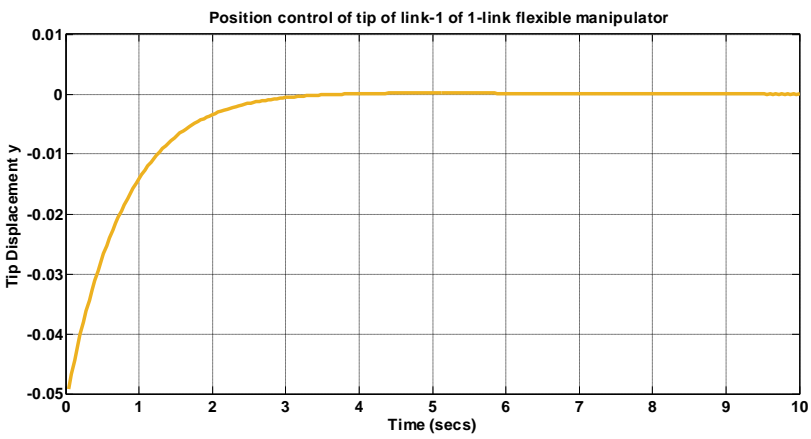

Fig. 5 : Tip position control setting to the track point, equilibrium point

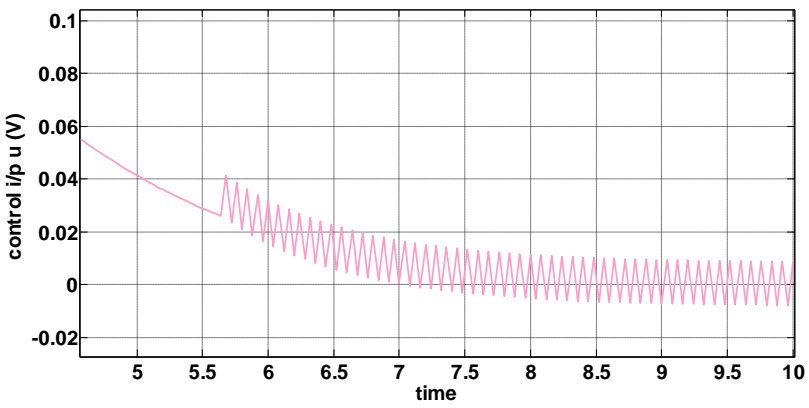

Fig. 6 : Control input plot showing the switching action

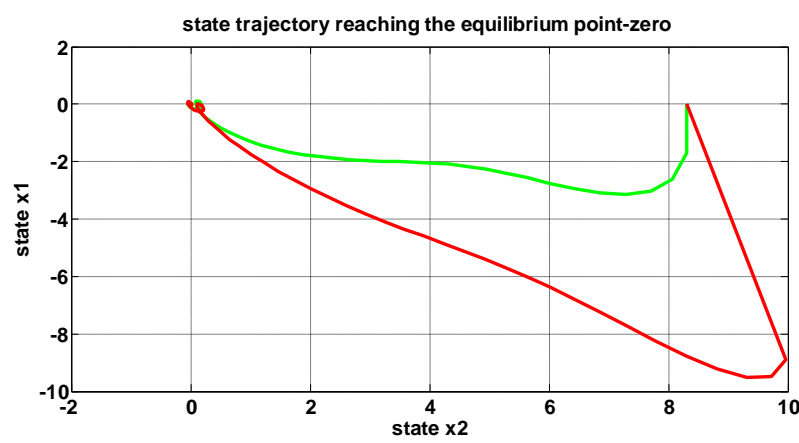

Fig. 7 : State trajectory (phase-plot) reaching equilibrium point of ' 0 ' showing tracking

From the Fig. 4/6, it can be seen how the chattering effect is seen, a zig-zag like motion \& finally from the Fig. 7, how the state trajectory (phase plot) reaches the equilibrium point (origin) from a given initial condition, say $x\left(t_{0}\right)$ can be seen. Also, from Fig. 5, it can be seen how the tip is controlled to a set-point (0).

\section{CONTROL DESIGN FOR DUAL LINK FLEXIBLE MANIPULATOR}

It should be noted that in this case, a dual / double / 2-flexible links are connected to obtain the overall plant structure so that the $1^{\text {st }}$ motor or the $1^{\text {st }}$ actuator is connected to the hub, which is termed as the base motor or base actuator to which the link 1 is connected and to the end of the link-1, another $2^{\text {nd }}$ actuator or the $2^{\text {nd }}$ motor is connected, which is termed as the link-2. Refer Fig. 15-17 (appendix) for the diagrammatic representation of the flexible system with its specifications in Table 1 (appendix). Our ROI (region of interest) is the tip of the link 2 , which is to be controlled. The actuator/s are actuated upon by a control effort $u_{1} \& u_{2}$ such that the desired set-points are reached.

In the sense, when both the link gets actuated, the motor/s as well as the flexible links starts vibrating. At the tip of the flexible link-2, one displacement sensor is placed, which is used to give the feedback to the system and could be used for tracking purpose. Also, at the tip of the link-1 another displacement sensor-1 is attached for feedback purposes. This displacement $x_{1}(\theta)$ of the link $1 \& x_{2}(\theta)$ of the link 2 could be considered as the state variables, which is used for tracking and bringing back to the desired position in no time as a result of which the entire system becomes a 2 input, 2 output system or a MIMO system. In other words, there are two actuators, viz., (motor 1)-base motor \& (motor 2)-shoulder motor. These displacement $x_{1}(\theta)=y_{1} \& x_{2}(\theta)=$ $y_{2}$ are nothing but the outputs of the 2-link flexible manipulator system.

Simulation study has been carried out for a particular designed set values of the DSMC parameters $q, \tau, N, \Delta, \varepsilon$. The simulation results can also be observed for different sets of parameters of $q, \tau, N, \Delta, \varepsilon \&$ computing the switching surfaces $s(k)$ along with the control inputs $u(k)$, which could be also seen that the responses of the system states $x_{1}, x_{2}$ converges to zero (0) from a given IC $x(0)$. Also, the phase plots for the flexible system shows that the system states exhibiting the quasi-sliding motion. Another reaction plot demonstrates 
that the exchanging capacity diminishes towards zero from the underlying worth and remain inside a little band in the area of the exchanging line. Since the single-link model is modelled as a $(4 \times 4)$ case, there will be only 2 states $x_{1}, x_{2}, x_{3} \& x_{4}$ as the size of the $A$ matrix will be of $(4 \times 4)$. The values chosen for the tracking control were

$$
q=2, \tau=0.4, N=20, \Delta=0.2, \varepsilon=0.01 .
$$

From the sim results, it can be clearly observed that the set-point tracking control is good. Matlab tool has been used to develop the.$m$ code. The developed algorithms are written in the form of a code, the code is run and the various responses that were considered in the sim study were observed, the results were discussed. The following parameters were observed from the simulation results.

- State trajectory

- Phase plots

- Output tip displacements

- System states

- Switching function

- Control input
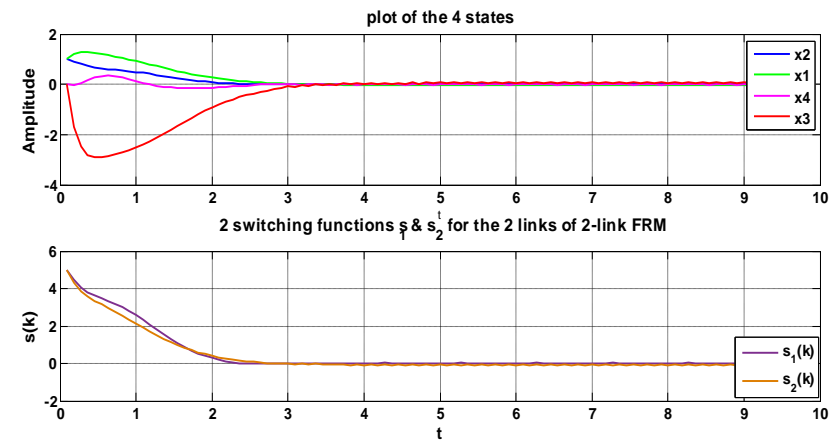

Fig. 8 : State plots $\&$ the switching planes of the entire 2-link combined flexible system
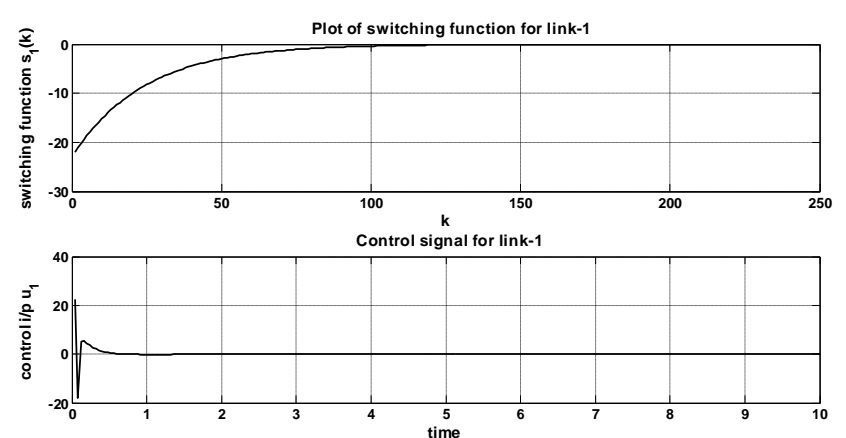

Fig. 9 : Plot of the control effort $u$ showing the switching action using the switching function $s(k)$ for the one link FRM

From simulation results of considered various previous parameters, it can be concluded that the effectiveness of the proposed algorithms are observed as they reach the stability point in no time (just within 5 seconds). From the frequency response plot, it can also be observed how the effect has taken place before $\&$ after the control effect (not shown here for the sake of convenience). The control effect taken is on the higher side compared to that of the 1-link manipulator case as the 2-link planar flexible manipulator has to bear the weight of link-1, link- $2 \&$ the 2 actuators.

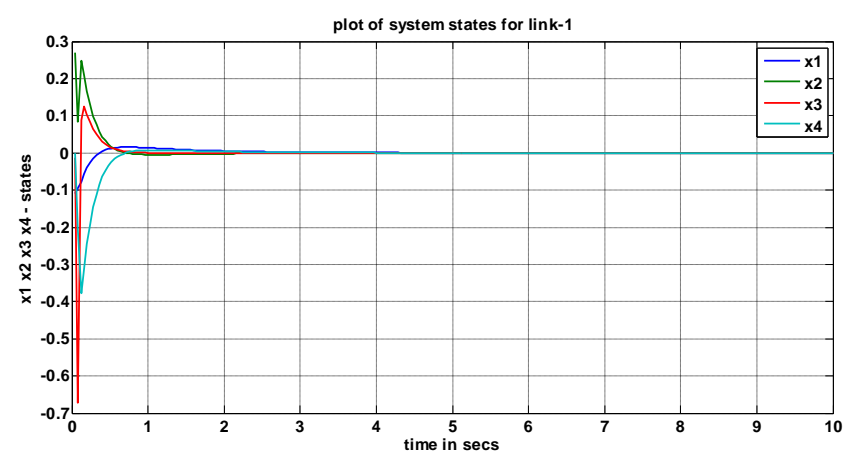

Fig. 10 : Plot of all the 4 system states for link-1 of FRM

From the Fig. 13, it can be seen how the chattering effect is seen, a zig-zag like motion \& finally from the Fig. 14, how the state trajectory (phase plot) reaches the equilibrium point (origin) from a given initial condition, say $x\left(t_{0}\right)$ can be seen. Also, from Figs. $11 \& 12$, it can be seen how the tip is controlled to a set-point ( 0 ) for the 2 links of the flexible manipulator.

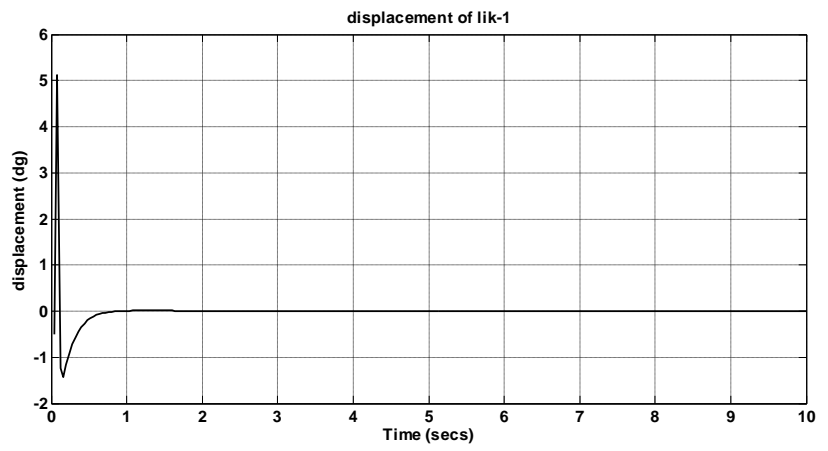

Fig. 11 : Tip displacement of the link-1 of the flexible manipulator

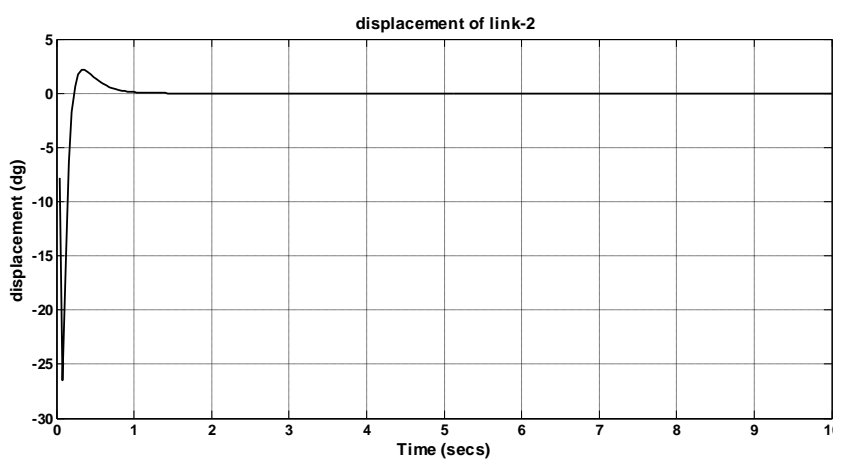

Fig. 12 : Tip displacement of the link-2 of the flexible manipulator
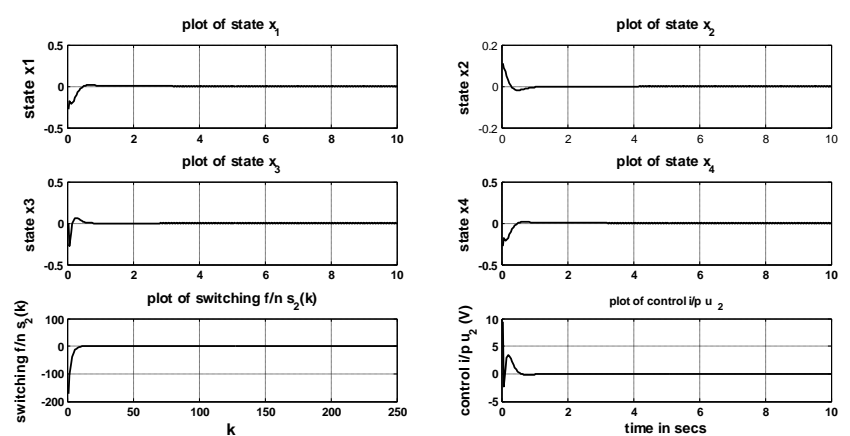

Published By:

Blue Eyes Intelligence Engineering \& Sciences Publication 
Fig. 13 : Plot of the system states, switching function \& control input required for the link- 2 of the flexible manipulator

The figure no. 10 depicts the graph of the 4 states of the system, which also shows that all the states reaches the equilibrium point.

\section{DEVELOPMENT OF THE DSMC SIMULINK MODEL}

The DSMC controller is also developed in the Matlab's environment with the simulink modelling in the background as shown in the algorithm of the flow-chart in the Fig. 2 for a 2-link case in which there are 2 sub-systems. It has to be noted that the model is the same for the 1-link case in which there will be one sub-system. The simulink model is constructed using sub-systems, sources, scopes, sinks, comparators, gain blocks, sample and hold circuits, multiplier blocks \& the connectors. All these mentioned blocks are available in the simulink modelling library.

Apart from these, various toolboxes such as control system tool box, optimization tool box, signal processing tool boxes available in the simulink library is being used. Various parameters are to be set in the different blocks that are used in the development of the simulink model. Simulation time taken is 0 to 10 seconds (varying-any time can be taken). For the discretization purposes, the time, i.e., sample time used in the simulation is $1 \mathrm{~ms}$. The developed simulink model is the same for both the types of flexible systems \& only the number of sub-systems will be different. The model is run for the given requisite simulation time $\&$ the same results obtained as shown in the Matlab Simulation outputs is observed for a 2-link case $\&$ the results are not shown here for a 1-link case here for the sake of convenience similar to the one presented in this research paper.

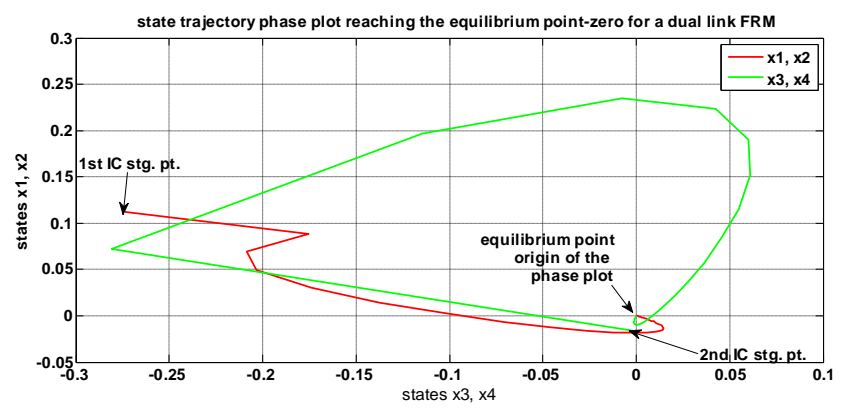

Fig. 14 : State trajectory (phase-plot) reaching equilibrium point of ' 0 ' showing tracking

\section{CONCLUSIONS}

Research was conducted on the set-point control, end-point displacements, control of the joints of the tip-1 \& tip-2 of the 1-link and 2-link FRM. The CT SS model of the 1 \& 2 link system was developed. Discrete sliding mode controllers using output samples were designed for the flexible manipulators to control the joint 1 of - link 1 of 1-link manipulator \& the joints $1 \& 2$ of - the 2 link flexible manipulator.

The developed .m file was run \& the simulation results were observed, in the sense the different simulation results (o/p's) are seen for the 2 FRM's considered singly. From the results of the Matlab simulation, it can be observed that when the system model's plant is put in loop with this proposed controller, the plant will definitely perform very well as seen from the simulated results in the figures. The output tip displacements reaches the set-point quickly in the form of the closed loop response performing much better than the open loop response.

The simulation results can also be observed for different sets of parameters of $q, \tau, N, \Delta, \varepsilon \&$ computing the switching surfaces $s(k)$ along with the control inputs $u(k)$. It can also be observed from the responses of the system states $x_{1}, x_{2}$ converges to zero (0) from a given IC $x(0)$. Also, the phase plots for the flexible system shows that the system states exhibiting the quasi-sliding motion. Another response plot shows that the switching $\mathrm{f} / \mathrm{n}$ will decay approximately to zero from an initial set value and stay in the neighbourhood of a small band in the neighbourhood of the line of switching.

It can also be observed for a 2-link case, the control effort needed for the link- 1 is more than that of the link- 2 as the base actuator has to control the weight of the link-1 + shoulder motor weight + the weight of the link-2 along with its end-effector payload mass, which can be justified from the control effort required for positioning diagrams in the simulation results.

In this article presented, a new DSMC control using MROF samples is being presented for a $n$-link FRM $(n=2)$. Further, it can also be observed that the DSM control law can be straightforwardly acquired as far as the yield tests $y$ and quick past control work $u$. The research work done is compared with some of the works of the yesteryears [21] [23]. Further, one main advantage of this methodology is that the calculation neither necessities the conditions of the framework for input reason nor for the exchanging capacity assessment \& therefore can be easily implementable in real time applications as the gains are piecewise constants.

\section{Appendix}

\begin{tabular}{|l|l|l|l|}
\hline $\begin{array}{c}\text { Material } \\
\text { properties }\end{array}$ & Symbol & Link-1 & Link-2 \\
\hline Length & $l$ & $15 \mathrm{~cm}$ & $10 \mathrm{~cm}$ \\
\hline Width & $w$ & $1 \mathrm{~cm}$ & $1 \mathrm{~cm}$ \\
\hline Thickness & $b$ & $0.1 \mathrm{~cm}$ & $0.1 \mathrm{~cm}$ \\
\hline $\begin{array}{l}\text { Moment of } \\
\text { Inertia }\end{array}$ & $I$ & $0.3619 \mathrm{E}-1$ & $0.3619 \mathrm{E}-1$ \\
\hline $\begin{array}{l}\text { Density } \\
\mathrm{kg} / \mathrm{m}^{3}\end{array}$ & $\rho$ & 0 & 0 \\
\hline $\begin{array}{l}\text { Young's } \\
\text { modulus } \\
\text { GPa }\end{array}$ & $E$ & 70 & 2.8 \\
\hline $\begin{array}{l}\text { Tensile } \\
\text { strength } \\
\mathrm{N} / \mathrm{mm}^{2}\end{array}$ & $R_{m}$ & 180 & 70 \\
\hline $\begin{array}{l}\text { Specific } \\
\text { strength } \\
\mathrm{mm} / \mathrm{kg}\end{array}$ & $R_{m} / \rho$ & 150 & 180 \\
\hline $\begin{array}{l}\text { Specific } \\
\text { stiffness } \\
\mathrm{Nm} / \mathrm{kg}\end{array}$ & $E / \rho$ & 25 & 25 \\
\hline
\end{tabular}


Table 1 : Specs of the 1-link \& 2-link flexible manipulator (length of link $2<\operatorname{link} 1$ )

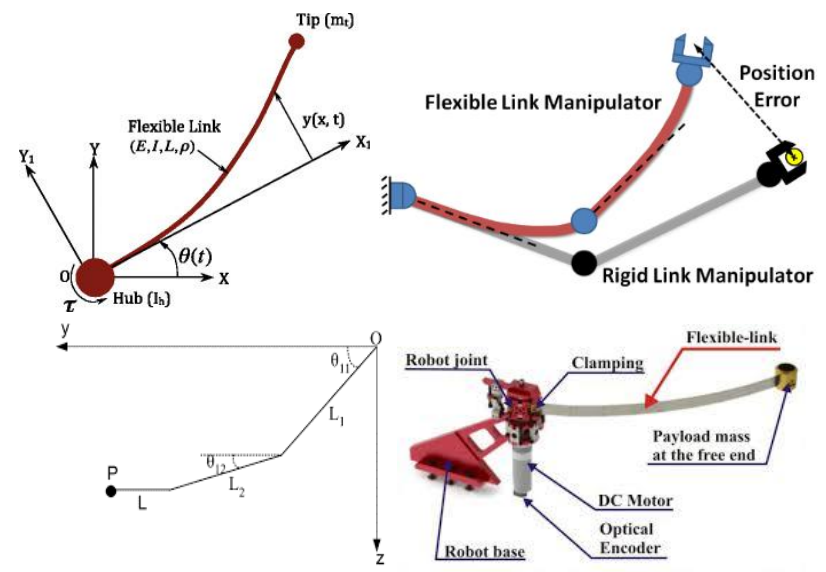

Fig. 15 : 1-link \& 2-link flexible robotic manipulators supported at the base

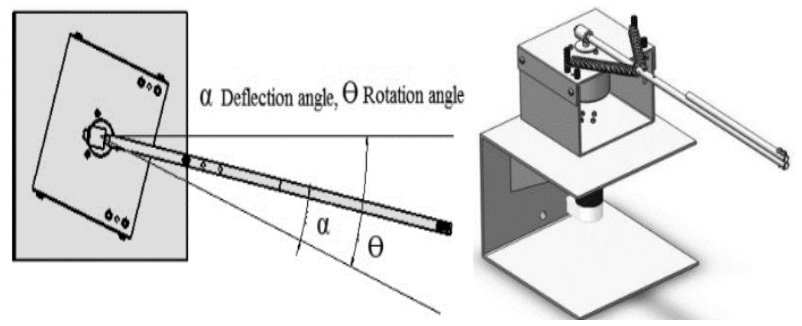

Fig. 16 : Single link flexible manipulator attached to the base motor
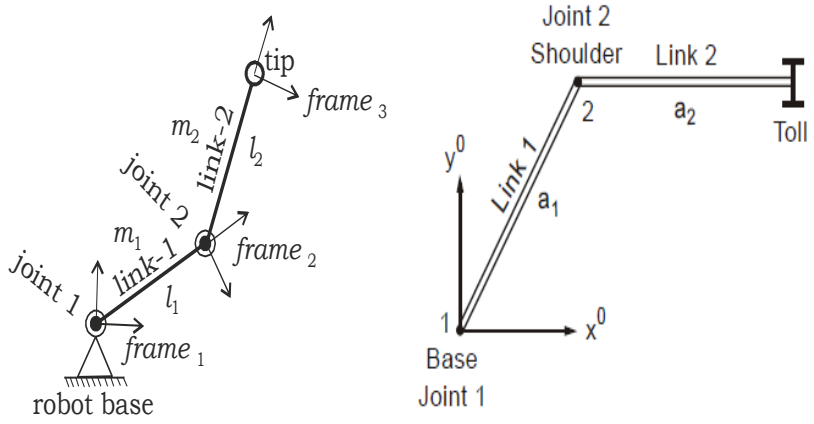

Fig. $17: 2$ link FM attached to the base motor-link $1 \&$ to the shoulder motor-link 2

\section{REFERENCES}

[1]. K. Desoye, P. Kopacek and P. Lugner, "Modelling of Flexible Robots - An Introduction", 2nd IFAC Symposium on Robot Control 1988 (SYROCO'88), Karlsruhe, Federal Republic of Germany, Elsevier's IFAC Proceedings, Vol. 21, Issue 16, Oct. 1988, pp. 21-28, 5-7 October 1988

[2]. Troch, Kopacek, "Control concepts and algorithms for flexible robots - An expository survey”, Symposium on Robot Control (1988), 2nd IFAC Symposium on Robot Control 1988 (Syroco '88), IFAC Symposia Series 1989, Selected Papers from the 2nd IFAC Symposium, IFAC Federal Republic of Germany, Karlsruhe, Volume 21, Issue 16, pp. 21-26 \& 29-34, 5-7 Oct. 1988.

[3]. Peng K.C. and Liou F.W., "A Survey of the Experimental Studies on Flexible Mechanisms", Flexible Mechanism, Dynamics, and Robot Trajectories, DE-VoI.24, ASME 21st Biennial Mechanisms Conference, Chicago, IL, pp.161-168, September 16-19, 1990.

[4]. Book W.J. \& Kwon, D.S., "Contact Control for Advanced Applications of Light Weight Arms", Journal of Intelligent and Robotic Systems, Vol. 6, No.1, August 1992, pp. 121-137.

[5]. Cannon R.H. Jr. and Schmitz E., "Initial Experiments on the End-Point Control of a Flexible One-Link Robot", The International
Journal of Robotics Research, Vol. 3, No. 3, pp. 62-75, Fall Oct. 1984.

[6]. Benosman and Vey G., "Control of flexible manipulators: A survey", Journal of Robotica, Vol. 2004, Cambridge University Press New York, NY, USA, pp. 533-545, 2004.

[7]. Thomas Looke, M. Bayoumi, Mohd. Umar Farooq, "Simulation of computed torque controllers for flexible manipulators", 34th Midwest Symposium on Circuits and Systems, Monterey, CA, USA, 14-17 May 1992.

[8]. Singh S.N. and Schy A.A., "Elastic Robot Control : Nonlinear Inversion arid Linear Stabilization", IEEE Transactions on Aerospace and Electronic Systems, Vol. 22, No.. 4, pp. 340-348, 1986

[9]. Inna Sharf, "Active Damping of a Large Flexible Manipulator with a Short-Reach Robot", Journal of Dynamic Systems Measurement and Control, Vol. 118, No. 4, pp. 3329 - 3333, DOI: 10.1109/ACC.1995.532220, American Control Conference, Proceedings of the Vol. 5, July 1995.

[10]. Menq C.H., J.S. Chen, "Dynamic modelling and payload-adaptive control of a flexible manipulator", IEEE International Conference on Robotics and Automation, Philadelphia, Print ISBN: 0-8186-0852-8, INSPEC Accession Number: 3228004, USA, pp. 488-493, 24-29 Apr. 1988.

[11]. Korolov V.V., Y.H. Chen, "Robust control of a flexible manipulator", IEEE International Conference on Robotics and Automation, Philadelphia, Print ISBN: 0-8186-0852-8, DOI: 10.1109/ROBOT.1988.12040, pp. 159-164, 24-29 April 1988.

[12]. S.P. Bhat \& D. K. Miu, "Precise Point-to-Point Positioning Control of Flexible Structures", Journal of Dynamic Systems Measurement and Control, Vol. 114, No. 3, pp. 416-421, Sep 01, 1992.

[13]. Jinjun Shan, Dong Sun, James K. Mills, S.K.Tso, “A PZT actuator control of a single-link flexible manipulator based on linear velocity feedback and actuator placement", Journal of Mechatronics, Vol. 14, No. 4, pp. 381-401, DOI: -7, May 2004.

[14]. Chen Y.P., Yeung K.S., "Regulation of a one-link flexible robot arm using sliding-mode technique", Int. Jr. of Control, Vol. 49, No. 6, pp. 1965-1978, 1989

[15]. P. Kotnik, S. Yurkovich, and U. Ozguner, "Acceleration feedback control for a flexible manipulator arm," J. Robot. Syst., Vol. 5, No. 3, pp. 181-196, 1988.

[16]. Bayo E., "Computed Torque for the Position Control of Open-Chain Flexible Robots", Proc. of the 1988 IEEE International Conference on Robotics and Automation, Philadelphia, PA, USA, pp. 316-321, Apr. 24-29, 1988.

[17]. J.M. Skowronski, "Algorithms for adaptive control of two-arm flexible manipulators under uncertainty", IEEE Transactions on Aerospace and Electronic Systems, DOI: 10.1109/7.9684, Vol. 24, No. 5 , pp. 562 - 570, Oct. 1988.

[18]. Xi Wang, Asok Ray, Peter Lee and Jinbo Fu, "Optimal control of robot behaviour using language measure", Int. J. Vehicle Autonomous Systems, Vol. 2, Nos. 3/4, pp. 147-167, 2004.

[19]. Ahmet S. Yigit, "On the Stability of PD Control for a Two-Link Rigid-Flexible Manipulator", Jour. of Dyn. Sys., Meas. \& Control, doi:10.1115/1.2899212, Vol. 116, No. 2, pp. 208 - 215, Jun 01, 1994.

[20]. Yao-Wen Tsai and Van Van Huynh, "Output Feedback and Single-Phase Sliding Mode Control for Complex Interconnected Systems", Hindawi's Jour. of Mathematical Problems in Engineering, Research Article (16 pages), Article ID 946385, Volume 2015, 2015.

[21]. B. Bandyopadhyay, V. Thakar, C. M. Saaj and S. Janardhanan, "Algorithm for Computing Sliding Mode Control and Switching Surface from Output Samples", The 8th Workshop on Variable Structure Systems - VSS2004, Vilanova I La Geltru, Spain, 6-9-2004 to 8-9-2004.

[22]. C. M. Saaj, B. Bandyopadhyay, and H. Unbehauen, "A new algorithm for discrete-time sliding mode control using fast output sampling feedback," IEEE Transactions on Industrial Electronics, vol. 49, no. 3, pp. 518-523, June 2002.

[23]. B. Bandyopadhyay and C. M. Saaj, "Discrete sliding mode control by non- dynamic multirate output feedback," Proceedings of seventh international workshop on variable structure systems, 2002, pp. 145-151. 
[24]. V. L. Syrmos, P. Abdalla, P. Dorato, and K. Grigoriadis, "Static output feedback: A survey," Automatica, vol. 33, no. 2, pp. 125-137, 1997.

[25]. A. Bartoszewicz, "Remarks on discrete-time variable structure systems," IEEE Transactions on Industrial Electronics, vol. 43, no. 1, pp. 235-238, 1996. 